\title{
An Empirical Exploration of a Neural Oscillator for Biped Locomotion Control
}

\author{
Gen Endo $^{1,2}$, Jun Morimoto ${ }^{2}$, Jun Nakanishi ${ }^{2,3}$ and Gordon Cheng ${ }^{2}$ \\ ${ }^{1}$ Sony Corporation, Tokyo, Japan \\ 2 ATR Computational Neuroscience Laboratories, Kyoto, Japan \\ ${ }^{3}$ Computational Brain Project, ICORP, Japan Science and Technology Agency, Kyoto, Japan \\ \{gendo, xmorimo, jun, gordon\}@atr.jp
}

\begin{abstract}
Humanoid research has made remarkable progress during the past 10 years. However, currently most humanoids use the target ZMP (Zero Moment Point) control algorithm for bipedal locomotion, which requires precise modeling and actuation with high control gains. On the contrary, humans do not rely on such precise modeling and actuation. Our aim is to examine biologically related algorithms for bipedal locomotion that resemble human-like locomotion. This paper describes an empirical study of a neural oscillator for the control of biped locomotion. We propose a new neural oscillator arrangement applied to a compass-like biped robot. Dynamic simulations and experiments with a real biped robot were carried out and the controller performs steady walking for over 50 steps. Gait variations resulting in energy efficiency was made possible through the adjustment of only a single neural activity parameter. Aspects of adaptability and robustness of our approach are shown by allowing the robot to walk over terrains with varying surfaces with different frictional properties. Initial results suggesting optimal amplitude for dealing with perturbation are also presented.
\end{abstract}

Keywords: Neural Oscillator; Central Pattern Generator (CPG); Biped Locomotion;

\section{INTRODUCTION}

Humanoid research and development has made remarkable progress during the past 10 years, for example: ASIMO (Honda) [1], SDR-4XII (Sony) [2] and HRP (AIST) [3]. Most of these presented humanoids utilize the target ZMP (Zero Moment Point) tracking algorithm for bipedal locomotion. ZMP is used to describe the stability of the system, and is used to control a system by following a target ZMP trajectory [4]. This control strategy provides a simple and straightforward method to permit biped robots to be realized. However, in order to accomplish successful locomotion this approach requires precise modeling and precise joint actuation with high joint control gains.

In many ways human walking is quite different to that of current biped robots. Humans change joint stiffness by activating antagonistic muscles depending on the phase of the walking cycle. Humans touch the heel to the ground first to absorb collision impact. The knee joint is extended in the middle of the stance phase to support the body weight mechanically exploiting a singular posture.

One of our goals is to develop biologically plausible bipedal locomotion algorithms. In doing so, we hope to gain a better understanding of the inner workings of human information processing. Our approach draws knowledge from related research areas such as neuroscience and biomechanics.

Here we will briefly introduce some related work: Passive Dynamic Walker (PDW) and CPG (Central Pattern Generator). Both strategies are closely related to achieving human-like walking.

A passive dynamic walker (PDW) can walk gently down a slightly inclined slope without any actuation; only gravitational force drives the robot. McGeer clearly showed that a walking motion can be realized intrinsically via mechanical design without any control input [5]. An efficient, natural, and smooth walking pattern was successfully demonstrated — resembling that of human. Like PDW, humans also exploit body dynamics to minimize energy expenditure during walking. Therefore, the idea of a passive dynamic walker will help us in deriving a controller that can produce effective human-like walking.

There is evidence showing that animals have inherent rhythmic pattern generators within their neural circuitry [6]. Many attempts have been made to investigate biped $\mathrm{CPG}$ controllers that have self-adaptive properties to cope with environmental changes. Taga successfully applied a CPG controller for an 8-link simulated planar biped model [7]. Antagonistic muscles for joint actuation were controlled using neural oscillators. In conjunction with appropriate neural connections and biologically based sensory feedback pathways - stable bipedal locomotion emerged. It is surprising that simple neural oscillators and their connections are adequate for bipedal locomotion. However, there is no analytical way to determine the parameters in the system; hand tuning is still required. Others have addressed this problem through parameter optimization [8][9].

Although many attempts have been made to investigate CPG controllers for legged locomotion, there are only a few successful experimental studies on actual robots. Noticeably Kimura's quadruped walker; it demonstrated amazing terrain adaptability and quick response against environmental changes [10]. Their system includes sensory feedback to the oscillators, such as foot tactile sensors and a body inclinometer. The framework demonstrated promising results of the $\mathrm{CPG}$ approach. It cannot be directly applied to biped locomotion but has provided hints toward realization of CPG controlled biped locomotion. 
In this paper, we investigate experimentally CPG controller for biped control based on current knowledge of biological systems. We address the problem of controlling a 5-link planar biped robot. Numerical dynamic simulation as well as experimental investigation is performed. The next section describes the biped model used. In Section III, the neural oscillator model and its arrangement are discussed. Section IV presents the CPG controller, implemented both on the dynamic simulator and on the real robot and compares experimental results. Additionally, the effectiveness of gait variation via changing the neural activity of the CPG is presented in Section V.

\section{BIPED MODEL}

Figure 1 shows our experimental system: A planar robot with lateral movement restricted by a boom to its left. A direct drive actuation was selected in order to control joint stiffness and provide back drivability. The knee joint is a wire driven system with reduction ratio of 2.0. Four motors are mounted in total. Feet with rounded soles are installed to allow rolling contact, and to exploit dynamics of the robot as in PDW. The foot has radius is $0.1[\mathrm{~m}]$ with a length of $0.06[\mathrm{~m}]$. Leg states (stance/swing) are detected by foot switches. Mechanical properties of the biped are shown in Table 1.

A numerical simulator was also developed for our studies. The simulator uses SD/FAST (Parametric Technology Corp, Inc.) for rigid body dynamics calculation. A simple springdumper model is employed to model reaction forces due to ground contact (see Appendix A).

Although effective for initial evaluation, we believe that numerical simulation is not sufficient to fully explore applicable bipedal locomotion. This is primarily due to the difficulty of collision modeling between feet and the ground, which directly affects the movements of the robot.
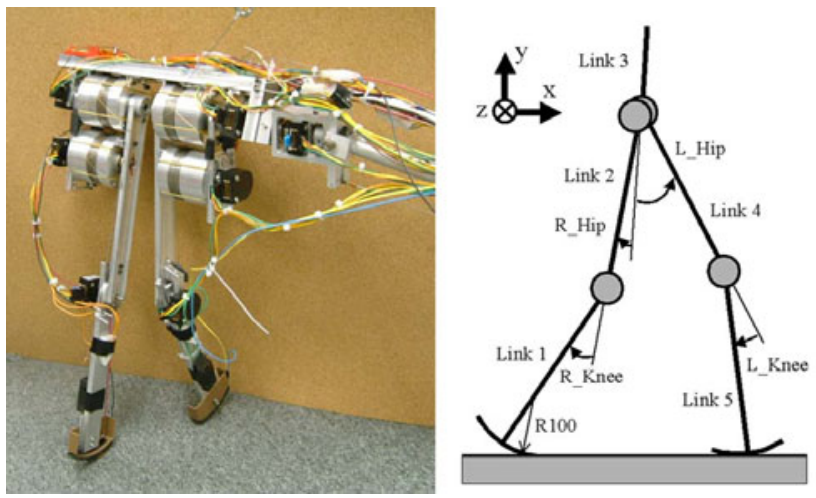

Figure 1. Left: physical system, Right: 5-link robot model

TABLE I. PHYSICAL PARAMETERS OF THE ROBOT MODEL

\begin{tabular}{|l|r|r|r|r|r|}
\hline & \multicolumn{1}{|c|}{ Link 1 } & \multicolumn{1}{|c|}{ Link 2 } & \multicolumn{1}{c|}{ Link 3 } & \multicolumn{1}{c|}{ Link 4 } & \multicolumn{1}{c|}{ Link 5 } \\
\hline mass $[\mathrm{kg}]$ & 0.05 & 0.43 & 1.00 & 0.43 & 0.05 \\
\hline length $[\mathrm{m}]$ & 0.20 & 0.20 & 0.01 & 0.20 & 0.20 \\
\hline inertia $\left(\times 10^{-4}[\mathrm{~kg} \cdot \mathrm{m}]\right)$ & 1.75 & 4.29 & 4.33 & 4.29 & 1.75 \\
\hline
\end{tabular}

\section{CPG CONTROLLER}

\section{A. Neural Oscillator}

The Neural Oscillator originally formulated by Matsuoka is commonly used to model CPG [11].

$$
\begin{aligned}
& \tau_{1} \dot{x}_{1}=c-x_{1}-\beta v_{1}-\gamma\left[x_{2}\right]^{+}-\sum h_{j}\left[g_{j}\right]^{+} \\
& \tau_{2} \dot{v}_{1}=\left[x_{1}\right]^{+}-v_{1} \\
& \tau_{1} \dot{x}_{2}=c-x_{2}-\beta v_{2}-\gamma\left[x_{1}\right]^{+}-\sum h_{j}\left[g_{j}\right]^{-} \\
& \tau_{2} \dot{v}_{2}=\left[x_{2}\right]^{+}-v_{2} \\
& y=\left[x_{1}\right]^{+}-\left[x_{2}\right]^{+}
\end{aligned}
$$

where $x_{1}, x_{2}, v_{1}, v_{2}$ are internal variables; $\tau_{1}, \tau_{2}, c, \beta, \gamma, h_{j}$ are constant parameters; $g_{j}, y$ are input and output signals respectively. Any number of inputs $g_{j}$ can be applied to the oscillator, which can either be proprioceptive signals, or signals from other neurons. As shown in Figure 2, the input is arranged to excite one neuron and inhibit the other, by applying the positive part $\left(\left[g_{j}\right]^{+}\right)$to one neuron and the negative part to the other. The inputs are scaled by a gain $h_{j}$ (Figure 2). Time constant $\tau_{1}$ and $\tau_{2}$ determine the output wave shape and its frequency. Neural activity $c$ represents tonic excitation modulating output amplitude. Without any input signals $g$, the neural oscillator can produce oscillation at its natural frequency via $\tau_{1}$ and $\tau_{2}$. If $g$ is large enough, and close to the oscillator's natural frequency, the phase difference between input and output is tightly locked due to entrainment. It is impossible to analytically solve the above equations. However Williamson has numerically investigated relationship between sinusoidal input and the oscillator's output in conjunction with the effect of the constant parameters [12], suggesting that an empirical exploration of CPG for biped locomotion is feasible.

\section{B. Neural Oscillator Arrangement}

Previous approaches follow a physiological arrangement, directly connecting the CPG to each joint to model antagonistic muscles and CPG outputs are used as torque commands. However, it is not necessarily true that one joint is driven by only a pair of antagonistic muscles, as in case of humans. Human's musculo-skeletal system is actuated by multiple redundant muscles. For example, hamstrings and quadriceps

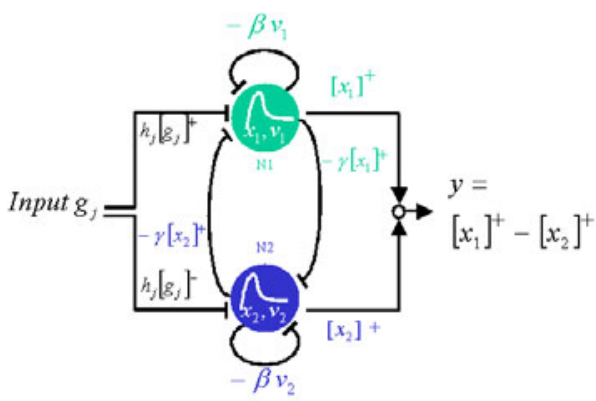

Figure 2. Matsuoka Coupled Neural Oscillator 
include double joint muscles that drive not only knee joint but also hip joint. Therefore, it may be natural to assume that some sort of signal conversion exists between CPG and muscles.

Additionally, in terms of development for applicable biped robot controller, this arrangement makes it difficult to understand how each oscillator contributes to the overall behavior, for example, the height of the center of gravity because of direct kinematics actuation. Furthermore, the neural oscillators are intrinsically non-linear. As a result, it is very difficult to adjust the parameters for the complete system based on a high-level criterion, such as the prevention of falling.

Inverse kinematics can be quite helpful for understanding the robot's movement in Cartesian space. A CPG could produce horizontal and vertical motion directly. Although Cartesian control is common practice in robotics, but it is hard to believe that low level neural oscillators in biology would operate using Cartesian coordinates. Moreover, inverse kinematics requires avoiding singular postures. This motivated us to propose a new CPG arrangement that could satisfy both biological plausibility and ease of comprehension. We arrange neural oscillators for a compass-like biped robot, decomposing leg movements into a linear motion (LM) controlling leg length and a swing motion controlling the leg angle (SM) (Figure 3).

The compass-like biped is commonly used to approximate human walking motion in biomechanics, and there exist simulation studies that show sinusoidal linear motion and passive swing motion, given appropriate initial conditions can generate open-loop biped locomotion. The reaction forces profile is quite similar to human walking [13]. Therefore, we consider the compass-like biped as a biologically plausible model.

Additionally, the model decomposes leg function into weight support and thrusting force generation. They provide an easy way to intuitively understand the robot's motion. In this paper, we assume the existence of a local controller between a CPG and a muscle that drives each joint depending on two distinctive leg functions: weight support and leg swing. In other words, CPG outputs are not utilized for joint level commands but as controlling leg functionality.

\section{Neural Oscillator Connection and Feedback Pathways}

We assumed that a single oscillator drives one decomposed motion (LM/SM) and the oscillator output is a position command for each movement. Right and left neurons are connected by mutual inhibition to generate symmetrical motion (Figure 3).

We explored feedback pathways for each oscillator to produce a walking pattern that would cope with the interacting environment. We decomposed walking motion into stepping motion in place, and swing motion before combining them to produce the overall walking motion. The stepping motion without propulsion is achieved by LM, performing leg lifting and body support alternately. If the leg swing motion cooperates with LM with the proper phase, walking motion is produced.

Figure 4 shows stepping motion in place and the experimentally derived feedback pathways. The body pitch oscillation was generated by LM via an inverse phase expansion and contraction. When both legs are in the stance phase, the body pitch angle is fed back to LM to prevent falling from occurring; this is done by adjusting the leg length. For example, if the body inclines forward then the length of the front leg increases and the hind leg decreases to generate a backward recovery movement. We introduce a "stability measure", as an indicator to switch feedback pathways because pitching angle arises an opposite activation for LM depending on foot placement. We approximate the stability measure by

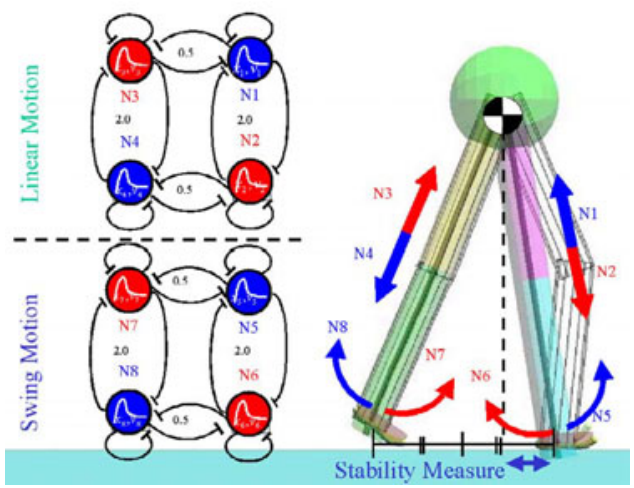

Figure 3. Compass-like biped and neural oscillator arrangement

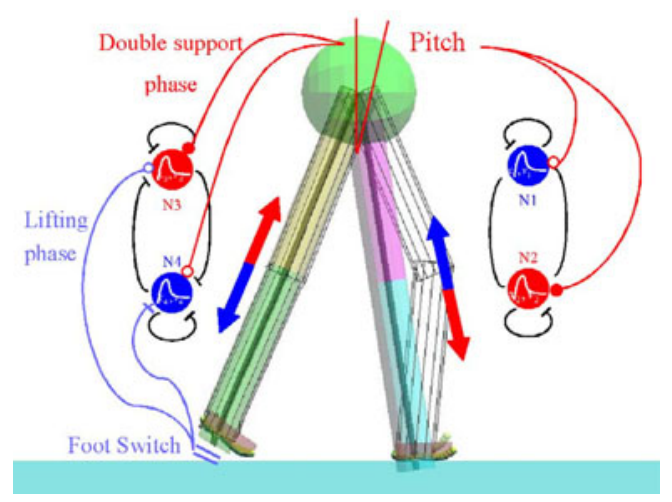

Figure 4. Feedback pathways for linear motion

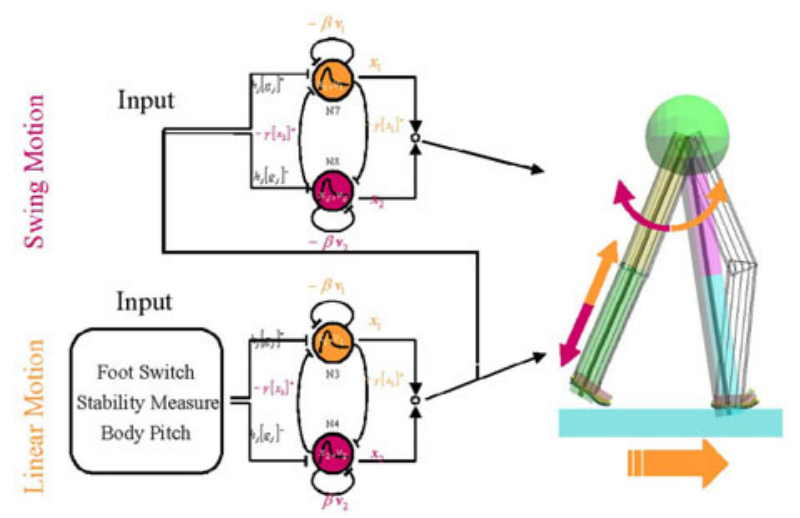

Figure 5. Feedback pathway for swing motion 
the distance between foot position and the projection of the hip joint to the ground (Figure 3). We also introduce an additional feedback pathway to provide clearance between the foot and the ground, in order to prepare for the swing motion. When the leg is lifted off the ground (detected by a foot switch), leg length is decreased.

We empirically examined the neural oscillators' parameters using the real robot and were able to achieve stable stepping motion that can cope with small disturbances. The parameter for the SM feedback pathway is also derived through experimentation, as shown in Figure 5. To induce cooperative movements LM output is directly fed into SM input. That is, SM takes forward maximum position with minimum leg length and takes backward maximum position with maximum length. This posture emulates the gravitational driving force of a PDW and provides forward falling motion. (see Appendix B for additional detail.)

(a)

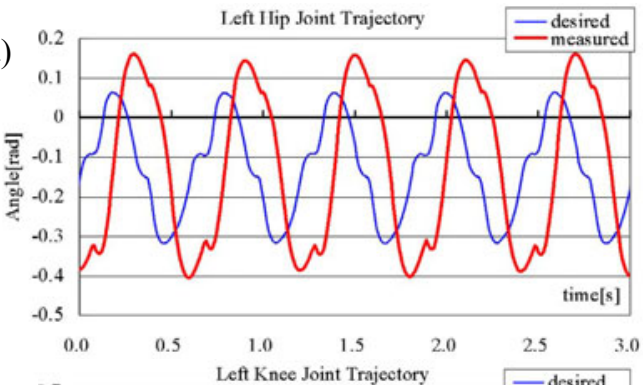

(b)

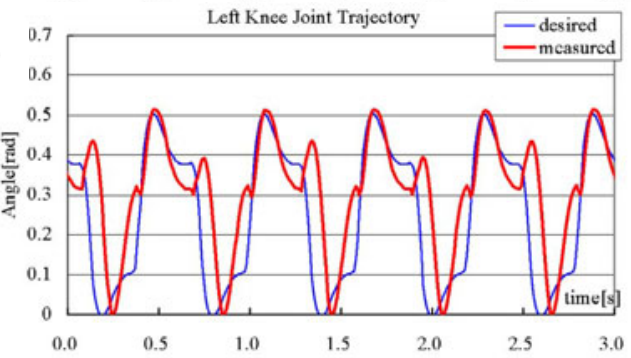

(c)

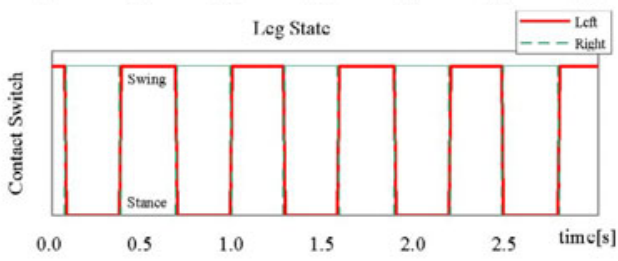

(d)

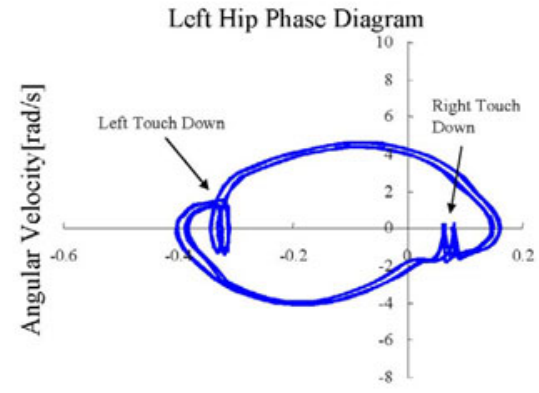

Angle[rad]

Figure 6. Simulation results (a) hip joint trajectory, (b) knee joint trajectory, (c) foot state, (d) phase plot of hip joint

\section{EXPERIMENT}

The controller was implemented on the real biped robot. At the beginning of every walking experiment, we suspended the robot to initiate appropriate gait with proper sensor signals. During 3-5 steps, oscillatory movements gradually entrained and a steady walking gait was established. The robot produced steady walking for over 50 steps with a typical walking period and velocity of $0.4[\mathrm{~s} / \mathrm{step}]$ and $0.47[\mathrm{~m} / \mathrm{s}]$.

The controller was implemented on both a dynamic simulator and on a real robot. Results of steady, constant walking are shown in Figures 6 (dynamics simulation) and 7 (experiment). The actual hip joint is entrained with constant phase delay compared to the neural oscillator output and the actual knee joint produced a large deviation from the desired posture when legs touched down. These errors resulted from low control gains. In terms of trajectory tracking control, these deviations indicate low joint accuracy. Conversely, the

(a)

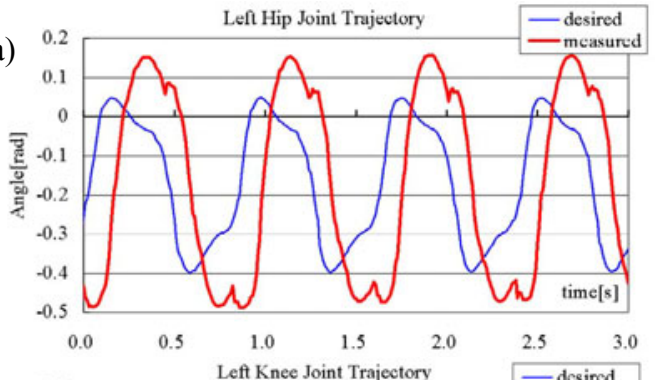

(b)

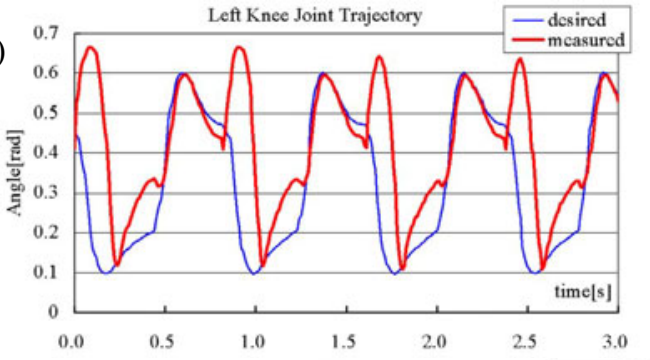

(c)

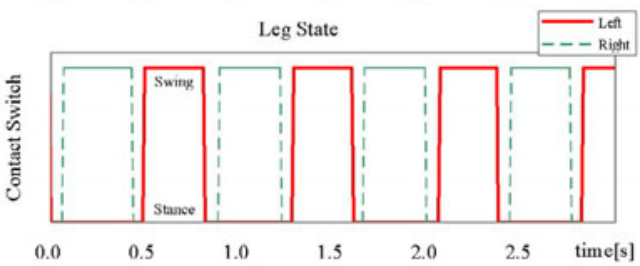

(d)

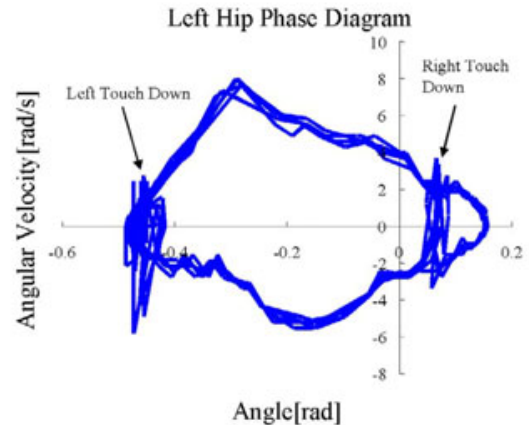

Figure 7. Experimental results: (a) hip joint trajectory, (b)knee joint trajectory, (c) foot state, (d) phase plot of hip joint 
controller effectively exploits the low gain with a virtual spring-dumper property to absorb the impact during leg touchdown. This result also indicates that walking movement is strongly related to joint gains.

Phase diagrams are shown in Figure 6 (d) and Figure 7 (d). They illustrate stable limit cycles for both simulation and the real robot. There are large angular velocity changes at leg touchdown, indicating that the relative vertical velocity between foot and ground is not zero. If joints are controlled rigidly to follow a nominal ZMP trajectory, the robot cannot cope with such a high frequency impact. As contemporary humanoids walk with unnaturally stealthy steps, producing motion that is unlike human motion. Humans effectively exploit heel strike to adjust walking velocity. Therefore, our controller is closer to human walking than ZMP. A large difference is shown between simulation and experiment during the touchdown phase. This is due to the difficulty of impact modeling in simulation.

With regard to step cycle, the real experiment shows a step cycle of 0.39 [s/step] whereas the natural frequency of the neural oscillator was $0.33[\mathrm{~s} / \mathrm{step}]$. This indicates that the step cycle was altered due to entrainment, thus exploiting body dynamics effectively.

Robustness during walking was confirmed via the dynamic simulation and the robot implementation. In the simulator, we applied a forward perturbation force at the body (link 3 ) for a duration $0.2[\mathrm{~s}]$ at random intervals and using a typical parameter set the controller could cope with up to 3.7 [N]. Robustness depends on the stride length in terms of the stability measure. Figure 8 shows the relationship between oscillator activity parameter $c$ in (1) and (3), which is proportional to stride and maximum allowable disturbance. Gait changes are discussed in the next section.

We also made walking experiments on different surfaces with different friction property (Figure 9, a metal sheet 2[mm] thick, a cork sheet $3[\mathrm{~mm}]$ thick and carpet). Photos were taken every half second. The controller could deal with this friction property changes without any problem. After walking through this area, perturbed gait pattern converged to a steady gait within 2 to 3 cycles due to neural oscillator entrainment, and the robot continued to walk steadily.

\section{GAIT VARIATION}

We observed step length variation through the experiment depending on initial condition. For example, sometimes the left leg step was larger than the right leg step. Even in this case, the robot walked steadily walking without falling over. We investigated gait variation to improve walking efficiency by changing $c$ and measured the resulting walking velocity and mechanical power consumption to derive walking efficiency measured by specific resistance $\varepsilon$ [15]. The smaller $\varepsilon$ indicates the higher energy efficiency.

We could modulate the parameter $c$ between 2.0 to 4.0 effectively. Walking failed outside of this range, as the smaller the $c$ value, the smaller the step length. Larger $c$ values result in greater touchdown impact, disturbing stability. Interestingly,

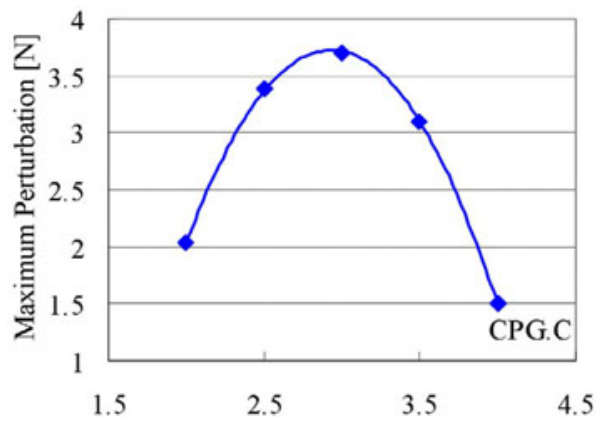

Figure 8 . Relationship between neural activity and maximum

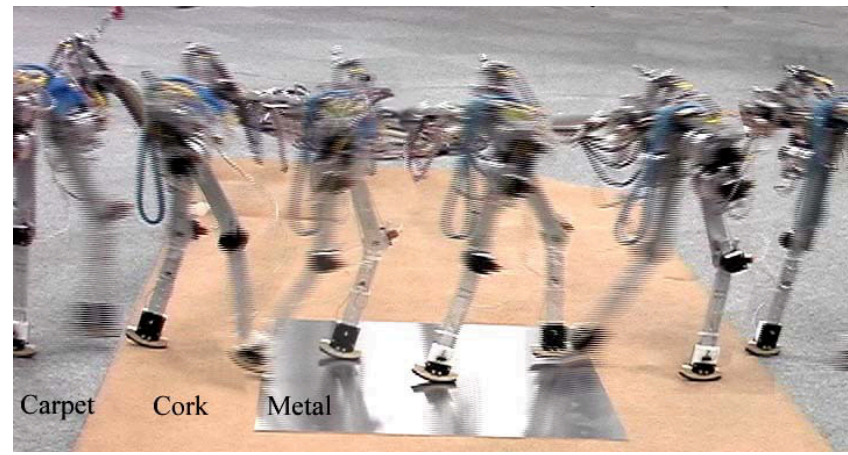

Figure 9. Walking on different surface

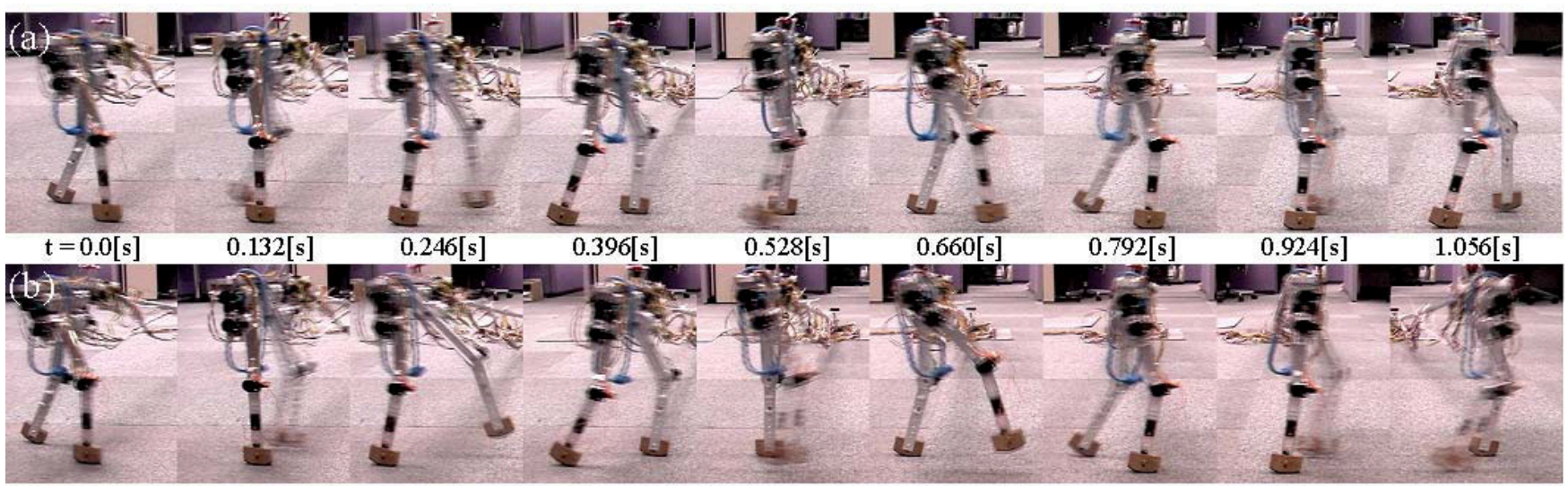

Figure 10. Gait variation: (a) $\mathrm{c}=2.0, \varepsilon=0.169$, (b) $\mathrm{c}=3.5, \varepsilon=0.294$ 


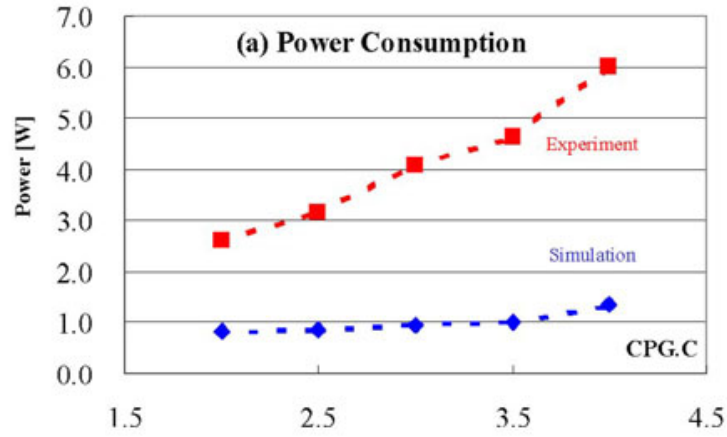

(b) Velocity and Specific Resistance $\varepsilon$

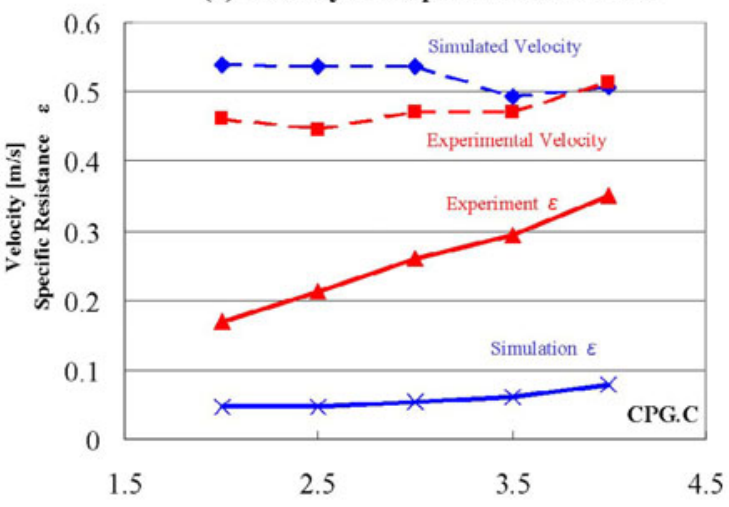

Figure 11. Neural activity and energy efficiency

(a) Power Consumption, (b) Velocity and specific resistance $\varepsilon$

we found that smaller $c$ gives rise to a more graceful walk and larger $c$ produces energetic marching like walk (Figures 10 a and b).

Figure 11 shows the relationship between $c$ and walking velocity, and the associated mechanical power consumption and specific resistance. Mechanical power consumption was calculated from the actuator torque command and the measured angular velocity.

Specific resistance $\varepsilon$ was obtained from power divided by measured velocity, mass, and gravitational acceleration. Experimental power consumption was 3-4 times higher than that of simulation. This is primarily due to inaccuracy of modeling of the impact. However, it was useful in providing an initial qualitative measure for our comparison.

Changing the neural activity $c$ did not significantly affect the walking velocity of the robot. It seems that the round foot shape and body dynamics actively contributes to walking velocity. Thus, walking velocity is determined by body dynamics, like a passive dynamic walker. Since this property is unavoidable for a dynamics dependent walker, different control strategies, such as posture control, may be needed to be investigated for slow walking, perhaps through ankle joint actuation. Larger neural activity $c$, required greater consumption of power. Minimum specific resistance was obtained at minimum $c=2.0$. However, the smaller step length decreased stability measure, so there is a trade-off between walking efficiency and stability. Thus, optimum $c$ is expected to be 2.0 to 4.0 , providing energy efficiency and stability.
Additionally, applied electrical power consumption was approximately $340[\mathrm{~W}]$ at $c=2.0$ and actuator efficiency was only $0.7 \%$. The same tendency was reported by the Spring Flamingo aiming at high efficient walking [16]. These results obviously show the importance of a careful hardware implementation in practice.

\section{CONCLUSION}

In this paper, we applied a biologically inspired walking algorithm to an actual experimental setup and compared between simulation and experiments. A neural oscillator controller was implemented on a planar biped. We proposed a new neural oscillator arrangement, for a compass-like biped model, that decouples leg length and leg angle control. Then neural connection and feedback pathways were investigated by empirical exploration and the controller implemented on a simulator and an experimental setup. We confirmed that the robot had a robust, steady walk and the simulation results showed similar tendencies as the experimental results. Finally, we discussed gait variations through neural activity that improve walking efficiency.

Currently we are studying velocity control by changing both the time constant and the neural activity. Though the range is not large, we experimentally verified velocity could be adjusted from $0.4-0.5[\mathrm{~m} / \mathrm{s}]$. Velocity largely depends on foot shape, so optimum radius and shape should be further investigated.

In this paper, we dealt with steady state walking and it was difficult to initiate walking. Transition controllers and posture controllers should be investigated in the future.

\section{ACKNOWLEDGMENT}

We would like to thank Seiichi Miyakoshi of the Digital Human Research Center, AIST, Japan for productive and invaluable discussions.

\section{APPENDIX A: GROUND CONTACT MODEL}

$x, y$ denote foot position, and $x_{g}, y_{g}$ are the ground contact points. Reaction forces are modeled as following.

$$
\begin{aligned}
& \text { if } y<y_{g} \\
& \qquad F_{x}=k_{x}\left(x_{g}-x\right)-b_{x} \dot{x}, \quad F_{y}=k_{y}\left(y_{g}-y\right)-b_{y} \dot{y} \\
& \text { if } y \geq y_{g} \\
& F_{x}=0, \quad F_{y}=0
\end{aligned}
$$

$F_{x}, F_{y}$ are the horizontal and vertical ground reaction forces, respectively. We set the horizontal spring coefficient $k_{x}=3000$, and dumping coefficient $b_{x}=10$. For the vertical direction, $k_{y}=30000$, and $b_{y}=100$. A slippage model is established using the following condition; if $F_{x}>\mu_{0} F_{y}$, then slip occurs. $\mu_{\sigma}=1.0$ is the static friction coefficient. 


\section{APPENDIX B: CPG PARAMETERS, FEEDBACK PATHWAYS, AND JOINT KINEMATICS}

Common parameters for all neural oscillators:

$$
\tau_{1}=0.6, \tau_{2}=1.2, \beta=2.5, \gamma=2.0
$$

Tonic excitation $c($ when $\mathrm{c}=2.05$, output amplitude $=1.0)$ :

$$
2.0 \leq c_{S W} \leq 4.0, \quad c_{L M}=c_{S W} / 3.0
$$

Neural connections for right-and-left inverse phase Figure 3

$$
\left\{\begin{array}{l}
h=0.5 \\
g_{\text {Left_SW }}=y_{\text {Right_SW }}, g_{\text {Right_SW }}=y_{\text {Left_SW }} \\
g_{\text {Left_LM }}=y_{\text {Right_LM }}, g_{\text {Right_LM }}=y_{\text {Left_LM }}
\end{array}\right.
$$

Feedback pathway for linear motion.

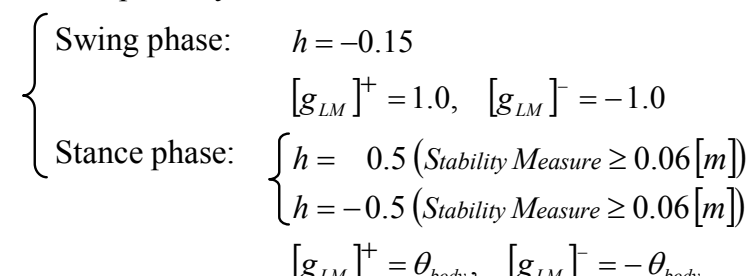

Feedback pathway for swing motion.

$$
\left\{\begin{array}{l}
h=0.5 \\
g_{\text {Left_SW }}=y_{\text {Right_LM }}, g_{\text {Right_SW }}=y_{\text {Left_LM }}
\end{array}\right.
$$

Relation of oscillator output and joint command (Figure 12)

$$
\begin{aligned}
& \theta_{\text {hip }}=-A_{S W} \cdot y_{S W}-A_{L M} \cdot y_{L M} \\
& \theta_{\text {knee }}=2 \cdot A_{L M} \cdot y_{L M} \\
& A_{S W}=4.0[\mathrm{deg}], \quad A_{L M}=13.5[\mathrm{deg}]
\end{aligned}
$$

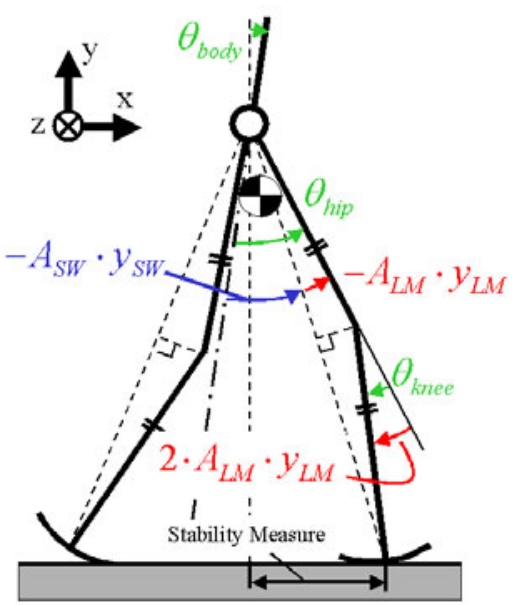

Figure 12. Oscillator output and joint command where $A_{S W}, A_{L M}$ are swing and linear motion amplitude, respectively. Servo gains are set as follows.

$$
P_{\text {gain }}=4.0[\mathrm{~N} \cdot \mathrm{m} / \mathrm{rad}], \quad D_{\text {gain }}=0.08[\mathrm{~N} \cdot \mathrm{m} \cdot \mathrm{s} / \mathrm{rad}]
$$

\section{REFERENCES}

[1] K. Hirai, M. Hirose, Y. Haikawa, T. Takenaka: The Development of Humanoid Robot, Proc. of Int. Conf. on Robotics \& Automation, pp.1321-1326 (1998)

[2] Y. Kuroki, M. Fujita, T. Ishida, K. Nagasaka, J. Yamaguchi: A Small Biped Entertainment Robot Exploring Attractive Applications, Proc. of Int. Conf. on Robotics \& Automation, pp.471-476 (2003)

[3] K. Kaneko, F. Kanehiro, S. Kajita, K. Yokoyama, K. Akachi, T. Kawasaki, S. Ota, T. Isozumi: Design of prototype humanoid robotics platform for HRP, Int. Conf. on Intelligent Robots and System, vol.3, pp.2431-2436 (2002)

[4] M. Vukobratovi'c, B. Borovac, D. Surla, Stoki'c: D. Biped Locomotion-Dynamics, Stability, Control and Application, SpringerVerlag (1990)

[5] T. McGeer: Passive Dynamic Walking, International Journal of Robotics Research, pp. 62-82 (1990)

[6] A. H. Cohen: Control principle for locomotion - looking toward biology, Proc. of 2nd International Symposium on Adaptive Motion of Animals and Machines, TuP-K-1 (2003)

[7] G. Taga: A model of the neuro-musculo-skeletal system for human locomotion I. Emergence of basic gait, Biological Cybernetics, vol.73, pp.97-111 (1995)

[8] K. Hase, N. Yamazaki: A Self-organizing model to imitate human development for autonomous bipedal walking, Proc. 6th International Symposium on Computer Simulation in Biomechanics, pp.9-12 (1997).

[9] M. Sato, Y. Nakamura, S. Ishii: Reinforcement Learning for Biped Locomotion, ICANN (2002)

[10] H. Kimura, Y. Fukuoka, T. Mimura: Dynamics Based Integration of Motion Adaptation for a Quadruped Robot, Proc. of 2nd International Symposium on Adaptive Motion of Animals and Machines, ThP-I-2 (2003)

[11] K. Matsuoka: Sustained oscillations generated by mutually inhibiting neurons with adaptation, Biological Cybernetics, 52, pp.345-353 (1985)

[12] M. Williamson: Neural Control of Rhythmic Arm Movements, Neural Networks, Vol. 11, Issues 7-8, pp.1379-1394 (1998)

[13] S. Miyakoshi, G. Cheng, Y. Kuniyoshi: Transferring Human Biped Walking Function to a Machine - Towards the Realization of a Biped Bike -, Proc. of 4th International Conference on Climbing and Walking Robots, pp. 763-770 (2001)

[14] G. Taga, Y. Yamaguchi, H. Shimizu: Self-organized control of bipedal locomotion by neural oscillators in unpredictable environment, Biological Cybernetics, vol.65, pp.147-159 (1991)

[15] G. Gabriell, T. von Karman: What Price Speed?, Mechanical Engineering 72, 775-781 (1950)

[16] J. Pratt: Exploiting Inherent Robustness and Natural Dynamics in the Control of Bipedal Walking Robots, Ph.D. Thesis, Computer Science Department, Massachusetts Institute of Technology, Cambridge, Massachusetts (2000) 